\title{
Solar Wind Composition Associated with the Solar Activity
}

\author{
X. Wang ${ }^{1}$, B. Klecker ${ }^{2}$ and P. Wurz ${ }^{3}$ \\ ${ }^{1}$ Key Laboratory of Solar Activity, \\ The National Astronomical Observatories, \\ CAS, Chaoyang District, Beijing \\ ${ }^{2}$ Max-Planck-Institut für Extraterrestrische, \\ Physik, Garching \\ ${ }^{3}$ Physikalisches Institut, Universität Bern, Bern \\ ${ }^{1}$ P.R. China \\ ${ }^{2}$ Germany \\ ${ }^{3}$ Switzerland
}

\section{Introduction}

The studies of elemental abundance of solar wind ions allows to address by open questions in several major fields of research: solar physics, heliospheric and planetary physics, and astrophysics and cosmology. This chapter is intended to provide the current status of knowledge about the solar wind composition mainly in relation to the solar physics with the emphasis on the effects of solar magnetic field.

The composition of the solar wind is mainly determined by the composition of the source material at the solar surface, and then modified by plasma processes in the solar atmosphere, operating in the transition region and in the inner corona. In recent decades, attention in composition studies has shifted from its early models toward differences in chemical fractionation as well as considerable fine-structure in the region above the solar surface. In-situ measurements of the solar wind composition give a unique opportunity to obtain information on the isotopic and elemental abundances of the Sun (e.g. Bochsler, 1998).

The magnetic field on the surface and in the atmosphere of the Sun is considered by many to play a significant role in the plasma processes, which is reflected in composition changes. The magnetic field on the solar surface includes two components: open magnetic flux, which opens into the heliosphere to form the heliospheric magnetic field (also called the interplanetary magnetic field); and closed magnetic flux, in the form of loops attached at both ends to the solar surface. The open magnetic flux controls many of the important processes in the solar corona. Reames (1999) argues that the interaction of loops with open flux is essential for an impulsive solar particle event, i.e., magnetic field reconnection causing the re-distribution of the magnetic field in the loop, the transfer of magnetic energy to the local plasma, and the escape of energetic particles. The interaction and reconnection 
between open flux and coronal loops releases matter and energy from the closed onto open field lines, which may add to the energetisation of the solar wind. As a result, open flux is broadly distributed on the Sun (Fisk et al. 1999; Fisk 2003; Fisk \& Zurbuchen 2006). The open flux also exhibits the reversal in polarity of the magnetic field over the Sun. The polarity of coronal mass ejection (CME) footpoints tends to follow a pattern similar to the Hale cycle of sunspot polarity. Repeated CME eruptions and subsequent reconnection will result in latitudinal transport of open flux and reverse the coronal fields (Owens et al. 2007). Understanding how the open flux of the solar surface behaves, how it is transported and distributed, is important for understanding the heliospheric plasma flow and the interplanetary magnetic field. The distribution of open flux can thus also be a sign of solar activity.

Solar wind charge states are indicative of the coronal electron temperature when assuming local thermodynamic equilibrium between the electrons and ions (Arnaud \& Raymond 1992; Bryans et al. 2006). Each charge state pair freezes-in at a different altitude, where the coronal expansion time scale overcomes its ionization/recombination time scale. Recent studies of charge states in fast streams found that the inferred electron temperature given by the in-situ observed charge states are higher than those derived from the spectroscopic measurements of the electron temperature. To resolve this discrepancy, two assumptions were adopted: one is assuming a non-Maxwellian velocity distribution for electrons with a suprathermal tail in the near-Sun region (Aellig et al. 1999; Esser \& Edgar 2000), the other is introducing the differential flow speed between the adjacent charge states of the same element with the assumption of the Maxwellian velocity distribution for the solar wind particles (Ko et al. 1997; Esser \& Edgar 2001). However, Chen et al. (2003) found that the differential flow speed has no significant impact on the charge state distribution of most of the heavy ions. The only way to resolve this issue is to introduce a non-Maxwellian electron velocity distribution. As we know, the fast solar wind is accelerated by ion cyclotron waves possibly generated by the interaction and reconnection between open flux and small scale closed loops. Once ions are perpendicularly heated by ion cyclotron waves and execute large gyro-orbits, density gradients in the flow can excite lower hybrid waves through which electrons can then be heated in the parallel direction (Laming \& Lepri 2007). A weak temperature gradient can lead to the development of non-Maxwellian suprathermal tails on electron velocity distributions, invalidating the Spitzer-Harm theory (Scudder \& Olbert 1983). Therefore, solar magnetic field fluctuations might be a reason to cause the non-Maxwellian velocity distribution of electrons in the fast solar wind. But things would be different in the slow solar wind because slow solar wind plasma is believed to accumulate in closed loops for hours to days before being set free into the heliosphere. The ions in the loops execute sufficient heating and their velocity distributions become almost isotropic. The conditions for exciting lower hybrid waves might not be satisfied because of the isotropic velocity distribution. Thus the charge state distributions in the slow solar wind would display different signatures from that in fast solar wind. In Section 2, we check the signatures of the solar magnetic activity on the charge states of heavy ions ( $\mathrm{Fe}, \mathrm{Si}, \mathrm{Mg}, \mathrm{Ne}, \mathrm{O}, \mathrm{C})$ in the slow and fast solar wind using ACE solar wind data, the "current sheet source surface" (CSSS) model of the corona, and SOHO MDI data during the 23rd solar cycle.

It is well established that the relative abundances of elements in the solar corona, solar wind, and solar energetic particles are similar (Meyer 1985). However, when compared to the photosphere, the particle populations do show an elemental fractionation that is organized 
by the first ionization potential (FIP). This elemental fractionation, the so called FIP effect is widely discussed in the literature, and there exist several models that give account of it, for example Marsch et al. (1995), Arge \& Mullan (1998), Schwadron et al. (1999), and Laming (2004). These models even differ in terms of the underlying physical processes (Hénoux 1998), and at present there is no consensus about the actual mechanism responsible for the FIP fractionation happening in the solar atmosphere. Moreover, when looking in detail at the various particle populations they do show some differences in different domains, as demonstrated for Ca (Wurz et al. 2003a) and later for several other heavy elements (Giammanco et al. ApJ 2007). In particular, energetic particles often show severe fractionation in their elemental composition. On the other hand, low-speed CMEs are the most difficult to identify within the ambient low-speed solar wind (Reisenfeld et al. 2003). In many aspects, CME-related flow reflects most closely the low speed solar wind, except for the general enhancement of He. It is still an open question whether the CME-related solar wind needs to be considered as an independent type of flow, or whether a low-speed solar wind reflects a composition, produced by a multitude of small-scale CMEs, that dissolve in the inner corona to form a plasma stream, while only the large-scale CMEs preserve their plasma signatures out to distances of in-situ spacecraft observations and remain identifiable as independent events (Bochsler 2007). For example, the elemental composition during the passage of the January 6, $1997 \mathrm{CME}$ was found to be different from the interstream, i.e., slow solar wind, and from coronal hole, i.e., fast solar wind, observed before and after it, respectively, with a mass-dependent element fractionation (Wurz et al. 1998). Moreover, in a study of CME plasma composition in the velocity range from 390 to $520 \mathrm{~km} / \mathrm{s}$ a strong deviation in composition of heavy ions with respect to slow solar wind was found for each CME (Wurz et al. 2003b). Schwadron et al. (1999) demonstrated that wave heating can account for both a mass-independent fractionation and a bias of low-FIP elements in active region loops but not on continuously open field lines. Their model placed several constrains on the rate at which waves isotropicalize and thereby heat the species distributions, e.g. the spatial scale of the discontinuity must be smaller than the gyroradius of protons.

In the following we will examine what is the role of the solar magnetic fields and their temporal evolution on the FIP fractionation? In examining the physical processes that can account for the mass-dependent element fractionation, we will focus on the waves associated with an-isotropic ion distributions that would act more effectively on heavy ions than on protons. In the following we will include both quasi-stationary and intermittent solar wind and give the attention to the abundance variations associated with the solar magnetic effects.

The evolution of the open magnetic flux on the solar surface reflects the changing properties of the solar activity. The solar wind associated with the distribution of open flux is expected also to contain the information of solar activity. One fact is that large abundance enhancements are observed in the solar corona over open magnetic field structures such as polar plumes (Feldman 1992; Sheeley 1996), and in active regions surrounding a sunspot with diverging magnetic field lines (Doschek 1983). Another factor is based on the recent models on impulsive events, a significant fraction of heavy elements that reside on the actively flaring flux rope is energized, the resonant interaction operates mainly on heavy elements with charge states increasing systematically with energy (Möbius et al. 2003; Klecker et al. 2006; Kartavykh et al. 2007). We are therefore motivated to explore the effect of solar magnetic activity on the abundance variations of heavy ions in the solar wind plasma. 
In Section 3, we compare the elemental abundance ratios $\mathrm{Fe} / \mathrm{O}, \mathrm{Si} / \mathrm{O}, \mathrm{Mg} / \mathrm{O}, \mathrm{Ne} / \mathrm{O}$, and $\mathrm{C} / \mathrm{O}$ over the $23 \mathrm{rd}$ solar cycle, where attention is being given to the varying fraction of open magnetic flux on the visible side of the solar disc.

\section{Effects of solar magnetic activity on the charge states of minor ions of solar wind}

We extrapolate the photospheric magnetic field into a global heliospheric field by using the Current Sheet Source Surface (CSSS) model (Zhao \& Hoeksema 1995). This model uses Bogdan and Low's solution (Bogdan \& Low 1986) for a magnetostatic equilibrium to calculate the effect of large-scale horizontal currents flowing in the inner corona and, by introducing the cusp surface and the source surface, uses Schatten's technique (Schatten 1971) to calculate the effects of the coronal and heliospheric current sheets and volume currents. These currents maintain the total pressure balance between regions of high and low plasma density. To model the effects of volume and sheet currents on the coronal magnetic field, we divide the solar atmosphere into three parts, separated by two spherical surfaces, i.e., the cusp surface and the source surface (see Figure 1 of Zhao \& Hoeksema 1995). The inner sphere, called the cusp surface, is located approximately at the height of the cusp points of coronal streamers. Above the cusp surface the coronal magnetic field is open everywhere. The outer sphere, called the source surface, is located near the reference height identified in the Parker's model above which the radially directed solar wind totally controls the magnetic field (Parker 1958). The cusp point is not easily defined and probably varies from place to place. However, the estimates of the height of cusp points from different experiments range from below 1.5 solar radii to above 3 solar radii (Zhao \& Hoeksema 1995). For instance, typical coronal streamers in the K-corona are approximately radial structures extending beyond $1.5-2.0$ solar radii. This implies a wide height range for the interactions of open and close field lines to happen. Recently, Laming and Lepri (2007) pointed out that any heating mechanism for electrons between 1.5 and 3 solar radii needs to explain the discrepancy between the SUMMER measurements of coronal electron temperatures and electron temperatures derived from Ulysses/SWICS charge state data of heavy ions in the fast solar wind. If most of the cusp points are located within $1.5-3$ solar radii, it implies that waves generated by reconnections of open and closed magnetic flux will further power the solar wind beyond the point where it can be observed by SUMER, i.e., ions are heated by ion cyclotron resonant Alfvén waves and part of the ion energy then leaks to electrons through a collisionless process (Laming 2004). This would provide a reasonable explanation to the charge state issue in the fast solar wind (Laming \& Lepri 2007).

Alternatively, the potential field-source surface (PFSS) model can be used to model the coronal magnetic field from the observed photospheric magnetic field. The difference between the models is that the PFSS model is without currents and the CSSS model is with current sheet-currents. There are two essential advantages of the CSSS model over the potential field-source (PFSS) model: first, in the CSSS model the field lines are open but not necessarily radial at the cusp surface which includes the effects of streamer current sheets; second, the source surface in the CSSS model is placed near the Alfvén critical point. In-situ observations of the heliospheric magnetic field should be compared with the magnetic neutral line near the Alfvén critical point. The radial component of the heliospheric magnetic field is latitude-independent, as detected by Ulysses (Smith \& Balogh 1995), and 
can be taken as uniform on a spherical surface above 5 solar radii (Suess \& Smith 1996). However, the magnetic field distribution on the source surface obtained using the PFSS model is not uniform, which does not agree with the Ulysses observations of the heliospheric magnetic field (Poduval \& Zhao 2004). In addition, the CSSS model shows better prediction of solar wind and interplanetary magnetic field (IMF) polarity and intensity measured near the Earth's orbit than the PFSS model. The correlation coefficient between the observed interplanetary magnetic field and the calculated 27-day averages is 0.89 for the CSSS model, which is better than that of the PFSS model, which is 0.77 (Zhao \& Hoeksema 1995). To obtain a uniform magnetic field on the source surface, we set the source surface at 15 solar radii (Zhao, Hoeksema, \& Rich 2002) and the optimum cusp surface is determined by matching trial calculations of Carrington rotation-averaged open magnetic flux with in-situ solar wind speed.

SoHO/MDI daily magnetic field synoptic data are used to obtain the daily proportion of open magnetic flux on the front side (i.e., the Earth-ward side) of the photosphere (Fig. 1a) during the 23rd solar cycle. We define the open magnetic flux fraction alpha $=o p n f /(o p n f+c l s f)$,
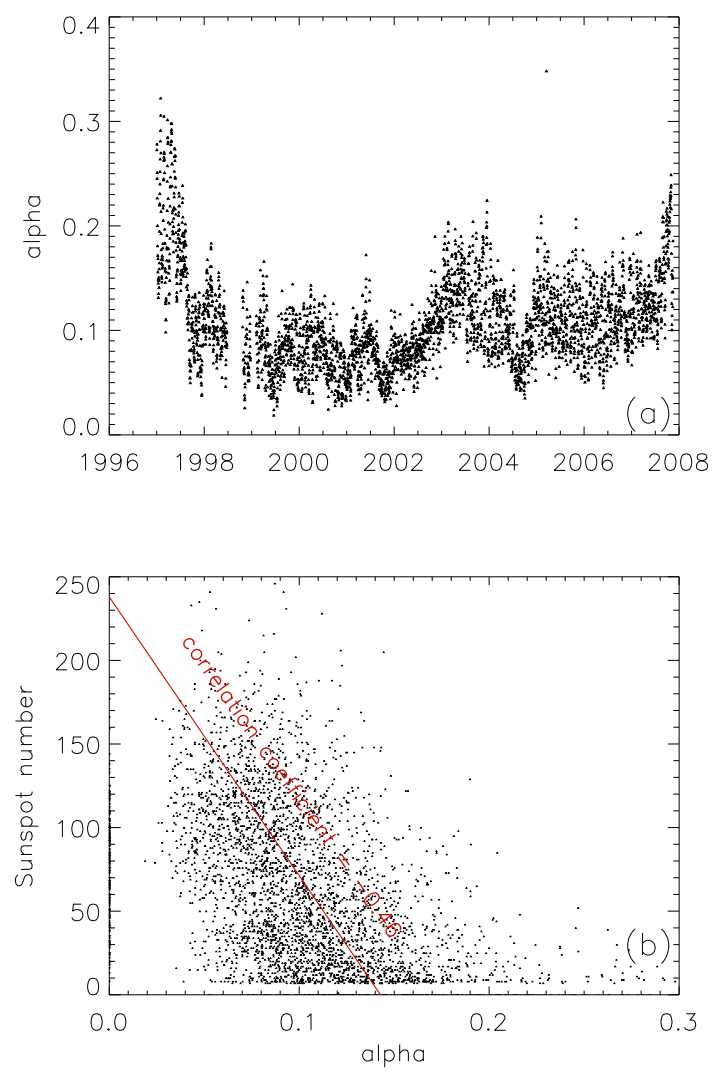

Fig. 1. (a) The fraction of the open magnetic flux on the front side of photosphere - alpha (for the definition of alpha see main body of the text) as a function of time during solar cycle $23^{\text {rd }}$. (b) The correlation of the daily fraction of open flux with the daily sunspot number. 
where opnf indicates open magnetic flux and clsf close magnetic flux, respectively, on the front side of the photosphere. The correlation of the open magnetic flux fraction alpha with the sunspot number is displayed in Fig. 1b. Thus, we can use the parameter alpha to describe the intensity of solar activity in this paper, where a small alpha corresponds to a high solar activity and vice versa..

The observed solar wind is traced back to the source surface in the corona along the Archimedian spiral assuming little radial acceleration (constant speed) and pure radial flow, neglecting interaction between fast and slow solar wind streams. That is, the heliographic latitude at the source surface is the same as that of in-situ observed point. The Carrington longitude at the source surface is shifted to the west according to the daily values of in-situ observed solar wind speed (Neugebauer et al. 2002). Here ACE/SWEPAM daily average solar wind data are used to infer the longitude shift. Once the shifted longitude is obtained, we get the time of day when the solar wind comes out of the source surface, and we further get the daily fraction of open magnetic flux on the front side of photosphere of that day. By this mapping technique, we can associate the in-situ observed solar wind with low, middle, and high solar magnetic activity, i.e., alpha $>0.14,0.075<a l p h a<0.14$, and alpha<0.075, respectively.

We do not use the inverse relation between flux tube expansion factor and solar wind speed, because the solar wind will be traced back to a region bounded within a narrow range of longitude that sensitively depends on the mapping speed used, but rather emphasize the influence of the background magnetic field on the properties of solar wind, i.e., the fraction of the open flux on the front side of the photosphere.

Note that the solar wind speed profile obtained at $1 \mathrm{AU}$ is the result of the interaction between solar wind streams of different speeds as they propagate outwards since the stream-stream interactions are inevitable. Our constant speed assumption would inevitably introduce the longitude shift error and cause a possible error in the mapping of solar wind with solar activity. However, we use the daily synoptic MDI data for the mapping that weakens the influence of the longitude shift error to some degree. For instance, a longitude error of less than 13 degree would not change the mapping result.

We analyzed ACE/SWICS charge state distribution data of heavy ions, from $\mathrm{Fe}, \mathrm{Si}, \mathrm{Mg}, \mathrm{Ne}$, $\mathrm{O}$, to $\mathrm{C}$, from the years 1998 to 2007 . We used the criterion $\mathrm{O}^{+7} / \mathrm{O}^{+6}<0.8$ to separate interplanetary coronal mass ejections (ICMEs) from the quasi-stationary solar wind, which is based on the results by Richardson and Cane (2004). The corresponding charge state distributions of $\mathrm{Fe}$ and $\mathrm{Mg}$ of the solar wind for six different speed ranges are compiled in Fig. 2. The wide range of charge states of the measured distribution is due to a mix of sources in the solar wind. As the solar wind speed is increasing, two opposite trends are identified: for iron, a charge state peak shifts from $Q=9$ to $Q=10$ with a tail extending to $Q=20$; for magnesium, the charge state peak shifts from $Q=10$ to $Q=9$ with a tail extending to $Q=5$. Opposite trends for Fe and $\mathrm{Mg}$ may be due to the effects of resonant acceleration at high altitude in the corona, where the magnetic effects dominate and preferentially accelerate species with lower charge state (that is with higher $\mathrm{m} / q$ ) in the slow solar wind. The slow solar wind plasma is believed to accumulate in closed loops in the solar atmosphere for hours to days before being released into the heliosphere by magnetic field reconnection of closed with open flux. The heating will occur in the loops of the corona by the interaction of ions with MHD turbulence, i.e., magnetic fluctuations. If ions are heated 
Solar Wind without ICMEs

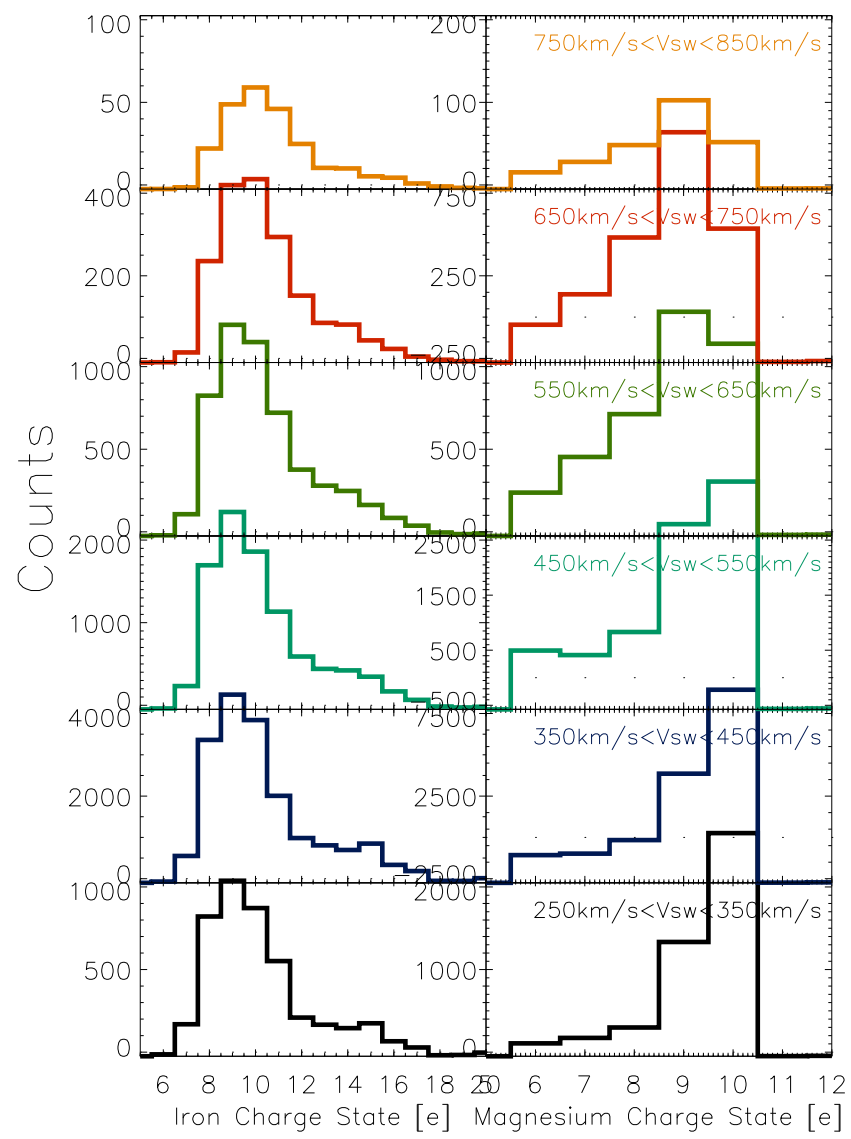

Fig. 2. Charge state distributions of Fe and $\mathrm{Mg}$ of the solar wind for six solar wind speed bins ranging from $250 \mathrm{~km} / \mathrm{s}$ to $850 \mathrm{~km} / \mathrm{s}$ during solar cycle $23^{\mathrm{rd}}$.

by magnetic fluctuations, which have a power-law spectrum $P(\omega) \propto \omega^{-\lambda}$, the ions with higher $m / q$ will be heated more strongly. Therefore, we see lower charge states of Fe ions in the slow solar wind. However, the $\mathrm{m} / \mathrm{q}$ for $\mathrm{Mg}$ ions is nearly half of that for Fe ions, which implies less power at the Mg-resonance. As a result, the resonant heating at high altitudes of the corona is much less efficient for $\mathrm{Mg}$ ions than for Fe ions. So the charge distribution of $\mathrm{Mg}$, dominated by $\mathrm{Mg}^{10+}$ over a wide range of electron temperatures, still keeps the information of the source temperature of the inner corona, with a different trend for the $\mathrm{Fe}$ charge states.

To explore the magnetic effects on the $m / q$ distribution, we calculated the mean charge states within different solar wind speed bins as a function of $m / q$, which are displayed in Fig. 3a. For comparison, the dependence on the first ionization potential (FIP) is given in Fig. 3b. We find that the fractions of the high charge states $\left(Q_{\mathrm{Fe}}>+10, Q_{\mathrm{Mg}}>+7, Q_{\mathrm{Si}}>+7, Q_{\mathrm{Ne}}>+7, Q_{\mathrm{O}}>\right.$ $\left.+6, Q_{C}>+5\right)$ increase with the solar wind speed when the $m / q$ is above 3; below this 


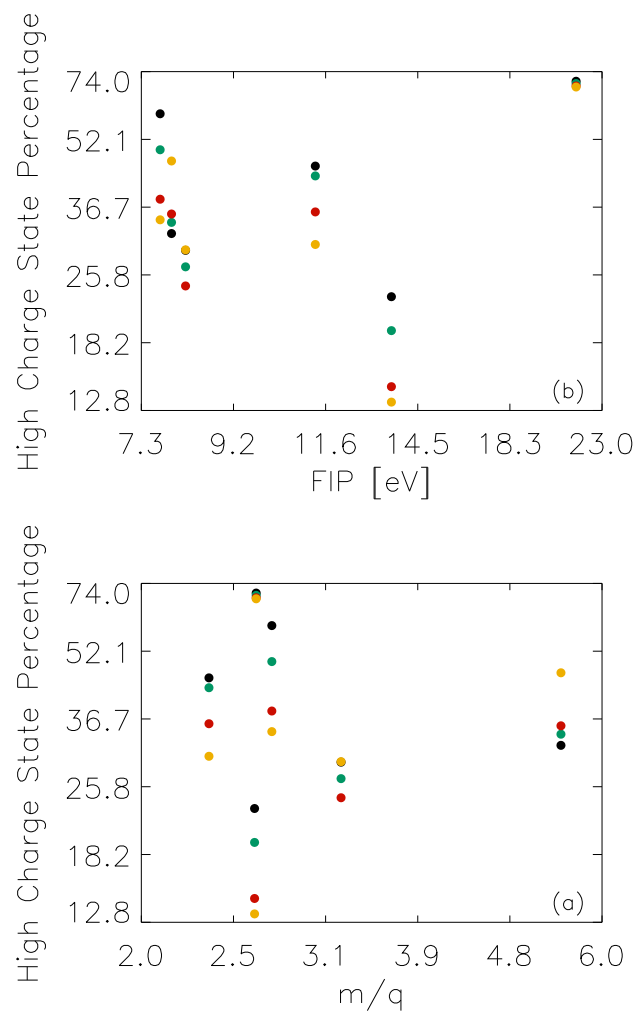

Fig. 3. (a) Mean charge states for four speed bins as a function of m/q (black: $250-350 \mathrm{~km} / \mathrm{s}$, green: $350-450 \mathrm{~km} / \mathrm{s}$, red: $450-550 \mathrm{~km} / \mathrm{s}$, yellow: $650-750 \mathrm{~km} / \mathrm{s}$ ); (b) Mean charge state for four speed bins as a function of FIP.

value, the fractions decrease with solar wind speed. No significant variation is found for $\mathrm{Ne}$ $(\mathrm{m} / \mathrm{q}=2.63)$. The resonant heating of ions by the magnetic fluctuation with a power-law spectrum will preferentially select species with lower charge state (i.e., with higher $\mathrm{m} / \mathrm{q}$ ) in the slow solar wind, which leads to the higher fractions of lower charge states in slow solar wind. On the other hand, ions are perpendicularly heated by ion cyclotron resonant Alfvén waves in the fast solar wind, electrons would be heated as well through the lower hybrid waves excited by the density gradients in the flow (Laming \& Lepri 2007). The increased electron temperature then further ionizes the plasma and leads to a higher ionization charge states in the fast streams. Once heated by lower hybrid waves, the electron distributions would depart from a Maxwellian velocity distribution (Laming \& Lepri 2007). This is consistent with the theoretical assumption of non-Maxwellian velocity distribution for electrons to solve the discrepancy on coronal electron temperatures associated with in fast streams (Aellig et al. 1999; Esser \& Edgar, 2000). In comparing the ionic charge states for different types of solar wind, Ko et al. (1999) found that the charge states of $\mathrm{C}$ and $\mathrm{O}$ for low latitude fast solar wind $\left(\mathrm{V}_{\mathrm{SW}}>500\right.$ $\mathrm{km} / \mathrm{s}$ ) are higher than those for south polar fast wind with the speed $\left(\mathrm{V}_{\mathrm{SW}}>700 \mathrm{~km} / \mathrm{s}\right)$, but the charge states of $\mathrm{Si}$ and Fe for low latitude fast wind are lower than those for south polar wind (see Figure 5 of their paper). This result does not violate the $\mathrm{m} / \mathrm{q}$-dependent response of 
the charge states to the magnetic effects in the fast solar wind, although the authors attributed the difference to either lower ion velocity or higher electron density toward lower latitude, rather than the electron temperature. They also compared the charge states for low latitude slow solar wind to those of south polar solar wind. But they did not find lower charge states for Fe in the slow solar wind (see Figure 6 of their paper) as we found in our observations. The discrepancy is likely due to either the latitude-dependence of the charge states in the fast solar wind or the possibility that the solar activity dependence of the charge states is more significant in low latitude than that in high latitude. At least near solar minimum this would be the case. Further investigation of this issue needs a combined data set observed from low latitude to high latitude.

The mean charge states for all the six heavy elements are compiled in Fig. 4, separated into groups of different solar magnetic activity, in which the black, green, red points correspond to the low, middle, high solar activity, respectively, as defined above. The speed intervals correspond to the ones indicated in Fig. 2. The error bar shows the 1-sigma statistical error of the mean charge state. When the solar activity is high, the error bars are large in the fast solar wind. However, for solar wind speeds below $700 \mathrm{~km} / \mathrm{s}$, the mean charge states still reveal a significant overall variation of the charge state distributions with solar activity. The yellow points in Fig. 4 correspond to the mean charge states, averaged over all three solar activity cases with a half speed interval of Fig. 2. When the solar wind speed is above 550 $\mathrm{km} / \mathrm{s}$, we find that the mean charge states depend significantly on the solar activity. At lower solar wind speeds, no significant solar activity dependence is found. Also, when plotted as a function of solar wind speed, the mean charge states for iron display a trend to increase with solar wind speed, and on the contrary, for magnesium display a trend to decrease with speed. For the other four ions $(\mathrm{Si}, \mathrm{Ne}, \mathrm{O}, \mathrm{C})$ this trend changes from negative speed dependence to positive speed dependence at the point near $675 \mathrm{~km} / \mathrm{s}$.

In conclusion, we present several interesting findings in this section: first, we observe a dependence of the charge state distribution of heavy ions with solar activity. This dependence is more important in the fast solar wind than that in the slow solar wind; second, iron is different from other species in that it displays lower charge states in slow wind than in fast wind; third, the fractions of the high charge states for Fe and $\mathrm{Si}\left(Q_{F e}>+10\right.$, $\left.Q_{S i}>+7\right)$ increase with the solar wind speed, while for the species with lower $m / q$, the fractions of the high charge states $\left(Q_{M g}>+7, Q_{O}>+6, Q_{C}>+5\right)$ decrease with the solar wind speed.

\section{Solar wind elemental abundances related to the Sun's open magnetic flux}

Using the CSSS extrapolation method introduced in Section 2, we analyzed the elemental abundance ratios $\mathrm{Fe} / \mathrm{O}, \mathrm{Mg} / \mathrm{O}, \mathrm{Si} / \mathrm{O}, \mathrm{Ne} / \mathrm{O}, \mathrm{C} / \mathrm{O}$, and $\mathrm{He} / \mathrm{O}$ as measured by ACE/SWICS, with attention given to the fraction of the open magnetic flux. In Fig. 5 we compiled the 3D plots for the charge states and elemental abundances versus solar wind speed for different alpha value. Each data point corresponds to a two-hour average. This survey covers the data from DOY 36, 1998 to DOY 110, 2007 of the ACE mission. The quality flags in the ACE data we used are quality flag $=0$ and 1 .

Mean abundance ratios and the charge state ratios relative to oxygen for the six solar wind speed bins are compiled in Fig. 6. Considering the ratios in Fig. 6, a systematic 


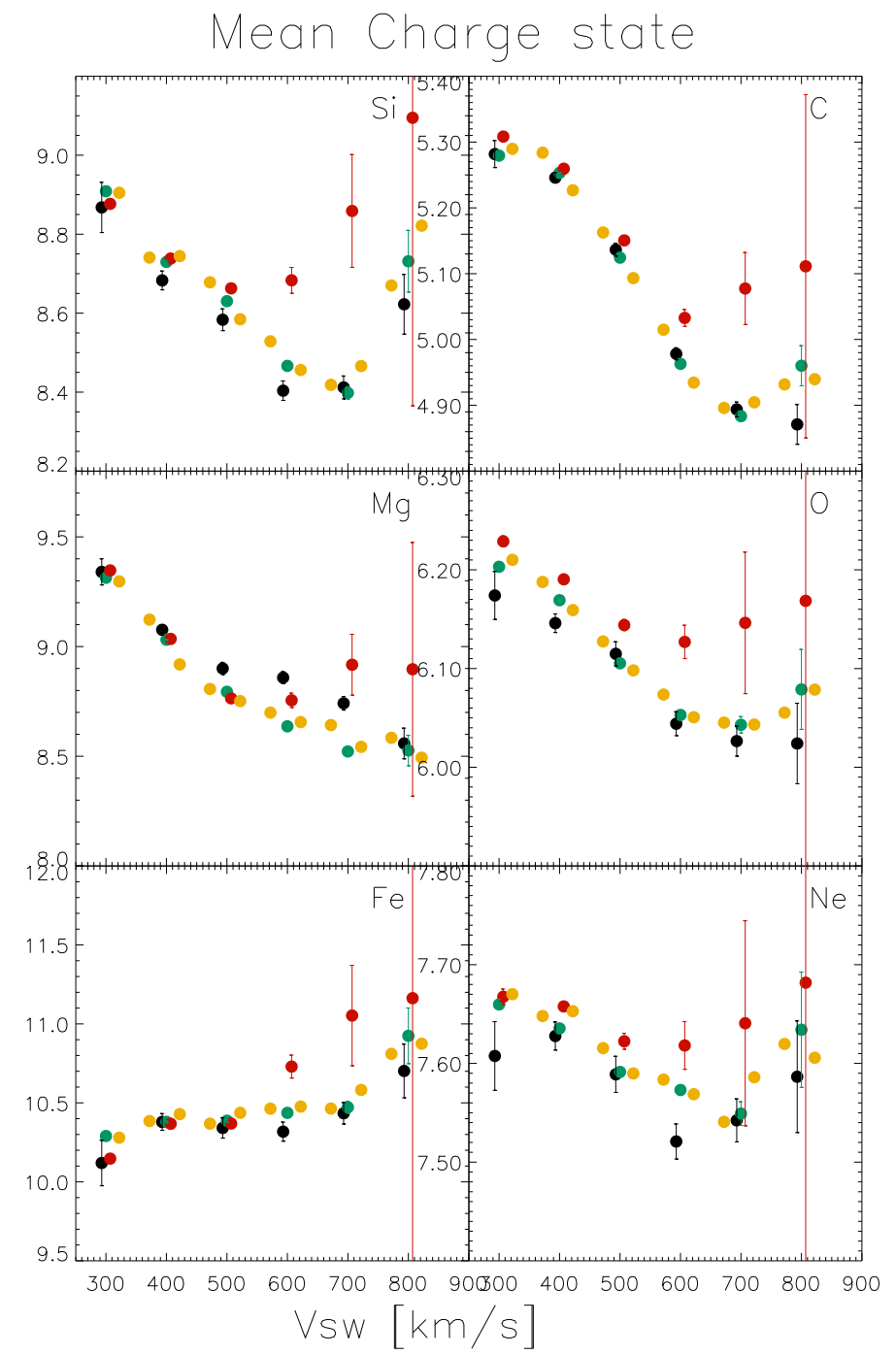

Fig. 4. Mean charge states for $\mathrm{Fe}, \mathrm{Si}, \mathrm{Mg}, \mathrm{Ne}, \mathrm{O}, \mathrm{C}$, underlying different solar magnetic activity (black - low activity, green - middle activity, red - high activity). Yellow points indicate the mean charge states of all solar wind bins without ICMEs.

instrumental error would only be a second order effect, the shown uncertainties are the statistical 1-sigma error. We find that, for the low-FIP elements $\mathrm{Fe}$ and $\mathrm{Si}$, the charge state ratios $\left(Q_{O} / Q_{F e}\right.$, and $\left.Q_{O} / Q_{S i}\right)$ in the high solar magnetic activity bin (alpha < 0.07$)$ are lower than the ratios in the higher activity bins when the solar wind velocity is between $550 \mathrm{~km} / \mathrm{s}$ and $750 \mathrm{~km} / \mathrm{s}$, and they tend to decrease with solar wind speed. Although this dependence is not obvious in another low-FIP element $\mathrm{Mg}$, the ratio $Q_{O} / Q_{M g}$ still shows some decreases in the high solar magnetic activity bin $(a l p h a<0.07)$ when the solar wind velocity is between 650 

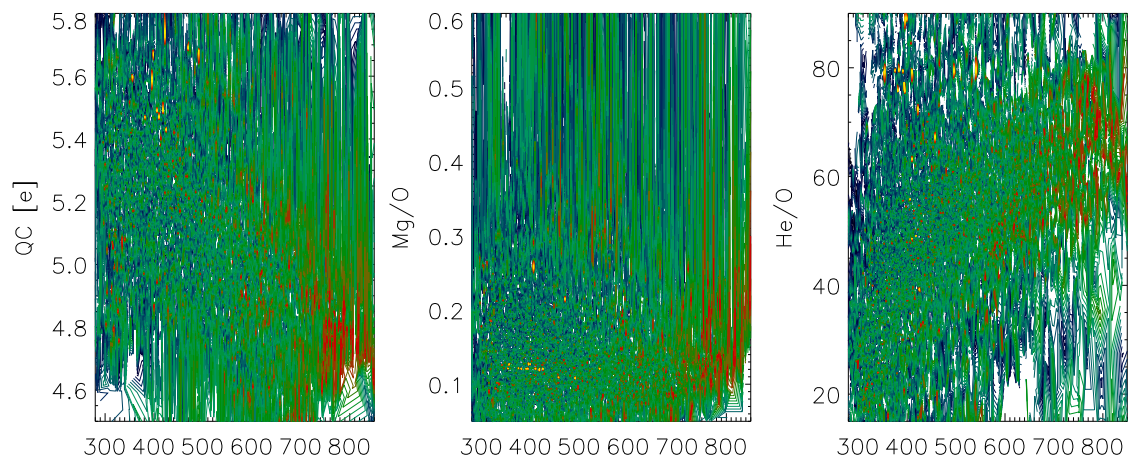

alpha
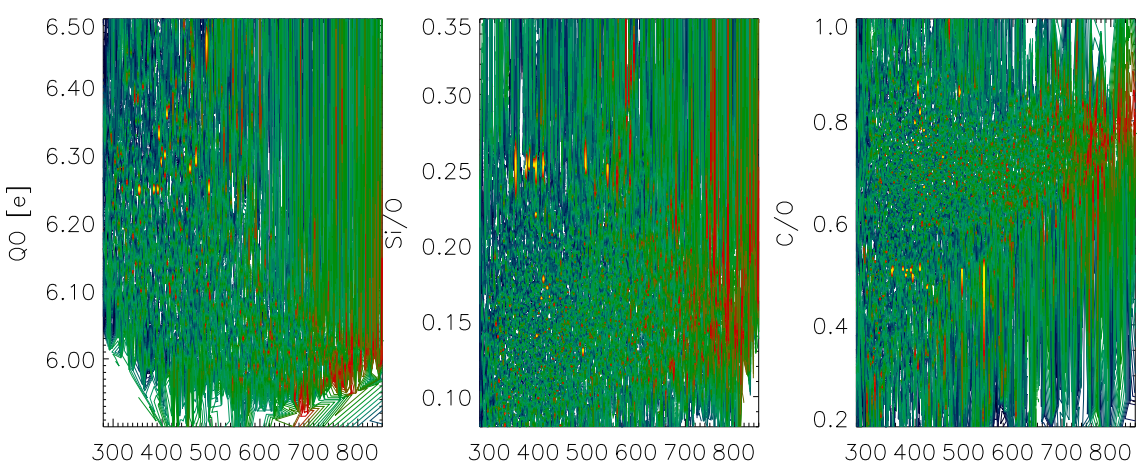

$20 \%$

$17 \%$

$14 \%$

$11 \%$

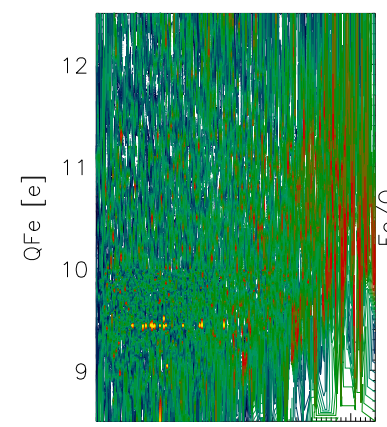

300400500600700800 Vsw $(\mathrm{km} / \mathrm{s})$
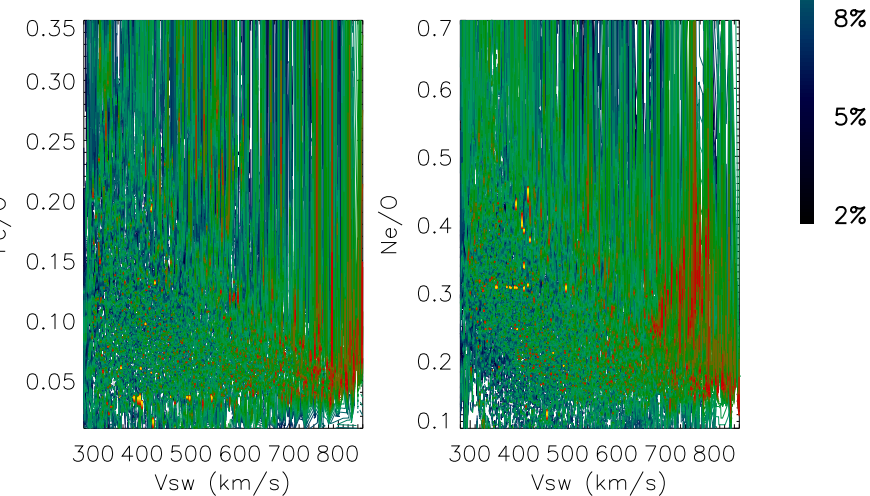

Fig. 5. 3D plots for the charge states and elemental abundance ratios versus solar wind velocity with different alpha. (alpha represents the fraction of the open magnetic flux on the visible side of the solar disc). $Q_{F e}, Q_{O}$ and $Q_{C}$ represent the charge states of iron, oxygen and carbon, respectively. Each point corresponds to a two-hour average, and the survey covers the ACE mission from DOY 36, 1998 to DOY 110, 2007.

$\mathrm{km} / \mathrm{s}$ and $750 \mathrm{~km} / \mathrm{s}$. For the high-FIP element neon, the ratio $Q_{\mathrm{O}} / Q_{\mathrm{Ne}}$ in the the high solar magnetic activity bin (alpha $<0.07)$ is found to be higher than the ratios lower activity bins when the solar wind velocity is between $450 \mathrm{~km} / \mathrm{s}$ and $650 \mathrm{~km} / \mathrm{s}$. For the mid-FIP element 

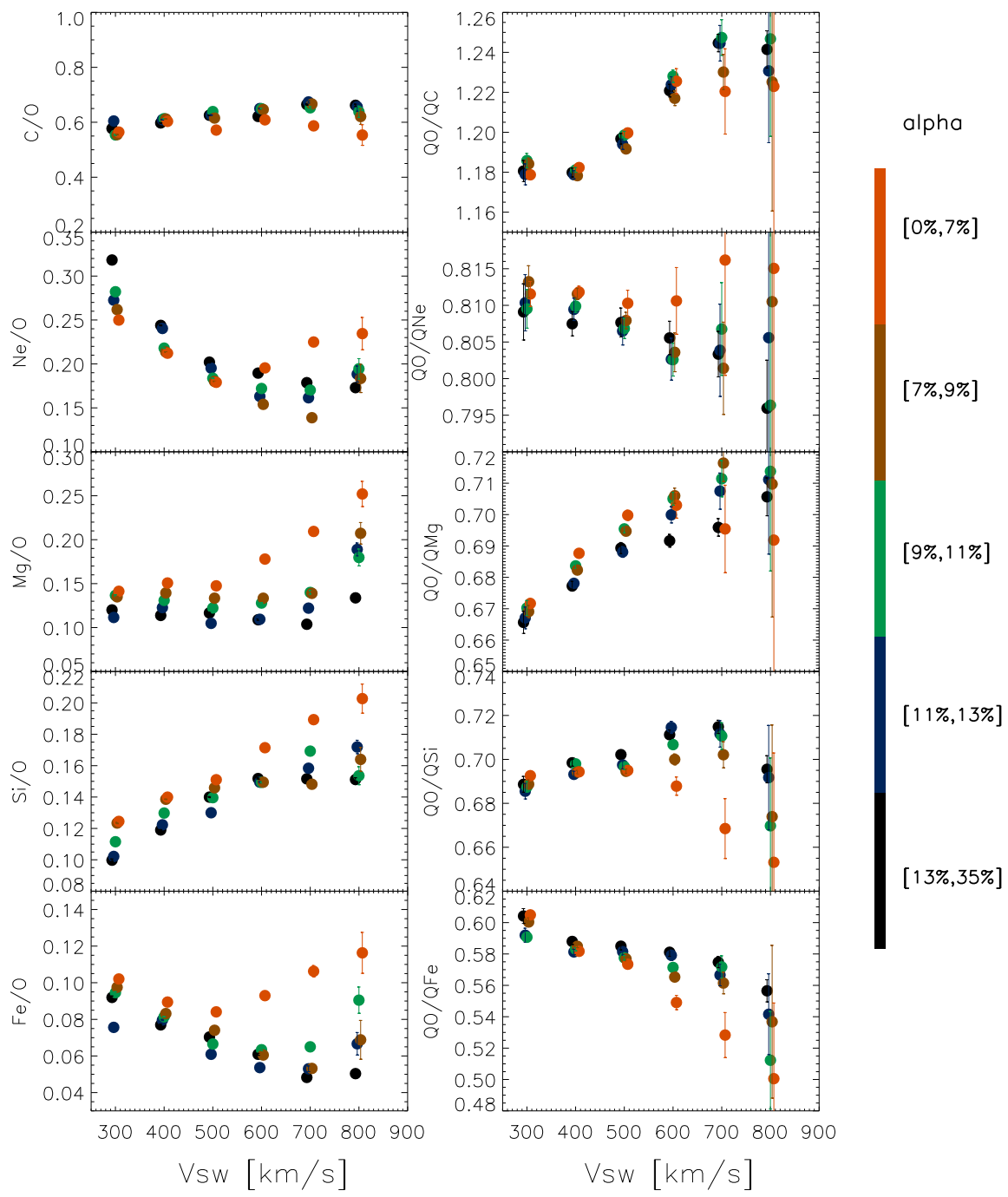

Fig. 6. Elemental abundance ratios and charge state ratios (relative to oxygen) versus solar wind speed for different alpha intervals. The error bars in the plot represent the statistical 1 sigma error of the mean.

carbon, the ratio $Q_{O} / Q_{C}$ does not show a significant variation between different activity bins.

In summary, the variation of charge state ratios with solar magnetic activity seems to be correlated with FIP. In order to show this correlation more clearly, we compiled in Fig. 7 the variation of charge state ratios in different solar magnetic activity bins as a function of FIP for the velocity bin $650 \mathrm{~km} / \mathrm{s}<\mathrm{V}$ sw $<750 \mathrm{~km} / \mathrm{s}$. The charge ratio, CR, indicates the ratio $Q_{O}$ / $Q_{X}$. It is clear that the ratio CR in the high solar magnetic activity bin $($ alpha $<0.07)$ to 


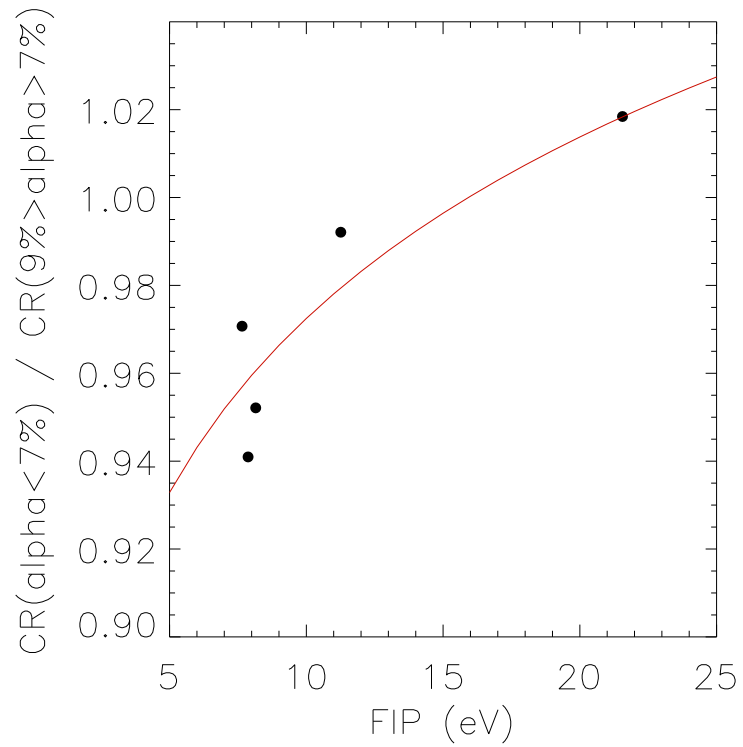

Fig. 7. Variation of charge state ratios in different alpha bins as a function of FIP for the velocity bin $650 \mathrm{~km} / \mathrm{s}<\mathrm{V}$ sw $<750 \mathrm{~km} / \mathrm{s}$, where $\mathrm{CR}$ indicates the charge state ratio $Q_{O} / Q_{X}$. The axis of ordinate is the ratio of CR in alpha $<0.07$ bin to CR in $0.07<$ alpha $<0.09$ bin, which increases from 0.94 to 1.02 as FIP is increasing. The red line shows a least-square fit to the data.

$\mathrm{CR}$ in the middle solar magnetic activity bin $(0.07<$ alpha $<0.09)$ bin exhibits a FIP dependence. We can fit this dependence with the empirical function $y=0.847^{*} \mathrm{FIP}^{* * 0.06}$, which is marked as red line Fig. 7. This dependence not only points to a non-negligible role of the configuration of background magnetic field in determining the charge states, indicating the importance of wave-particle interactions, it also suggests an important role of solar magnetic effects in the elemental fractionation of the solar wind.

We may note that both the charge state ratios and the abundance ratios in the slow solar wind do not show much variation between different solar magnetic activity bins. In the high solar magnetic activity bin $($ alpha $<0.07)$, the overall trend of the abundance ratios in the fast solar wind is clear: the abundance ratios rise with the solar wind velocity if the mass is above that of oxygen. When including a $10 \%$ to $20 \%$ measurement error for the ACE abundance data, the variation of $\mathrm{C} / \mathrm{O}$ with solar magnetic activity is lost and leads to a constant value of $\mathrm{C} / \mathrm{O}$ for all solar wind speeds. The ratio $\mathrm{Fe} / \mathrm{O}$ basically decreases with solar wind speed for low solar magnetic activity (i.e., when alpha is relatively high), which agrees with the results of previous work (e.g. Wurz et al. 1999; Aellig et al. 1999). The difference occurs for increasing solar magnetic activity, i.e., alpha is decreasing. As we know, CMEs, which are associated with high solar activity (Yashiro et al., 2004), can have large increases in the abundances of heavy elements, especially iron (Wurz et al., 1998, 2003b). These increases in elemental abundance are in addition to the enrichments of the FIP effect, where a factor 4 to 10 in enrichment for iron in the CME plasma is possible in individual events (Wurz et al., 1998; Wurz et al. 2003b). We show the distribution of the three types of 
solar wind and the fraction of CMEs in the five solar magnetic activity bins and six velocity bins in Fig. 8. For high solar magnetic activity (alpha < 0.07), the fraction of the CMEs increases from $1 \%$ to $71 \%$ as the solar wind speed increases from $300 \mathrm{~km} / \mathrm{s}$ to $800 \mathrm{~km} / \mathrm{s}$. If we make the statistical link between high solar wind speeds with the high fraction of CMEs during active times we can explain the enrichment of heavy ions for the fast solar wind in the low alpha bin. Statistically speaking, the correlation between solar activity and CMEs is well established (e.g. Hildner et al. 1976; Kahler 1992; Wang, Y. M. et al. 1998; Zhou et al. 2003; Gopalswamy et al. 2003; Yashiro et al. 2004).

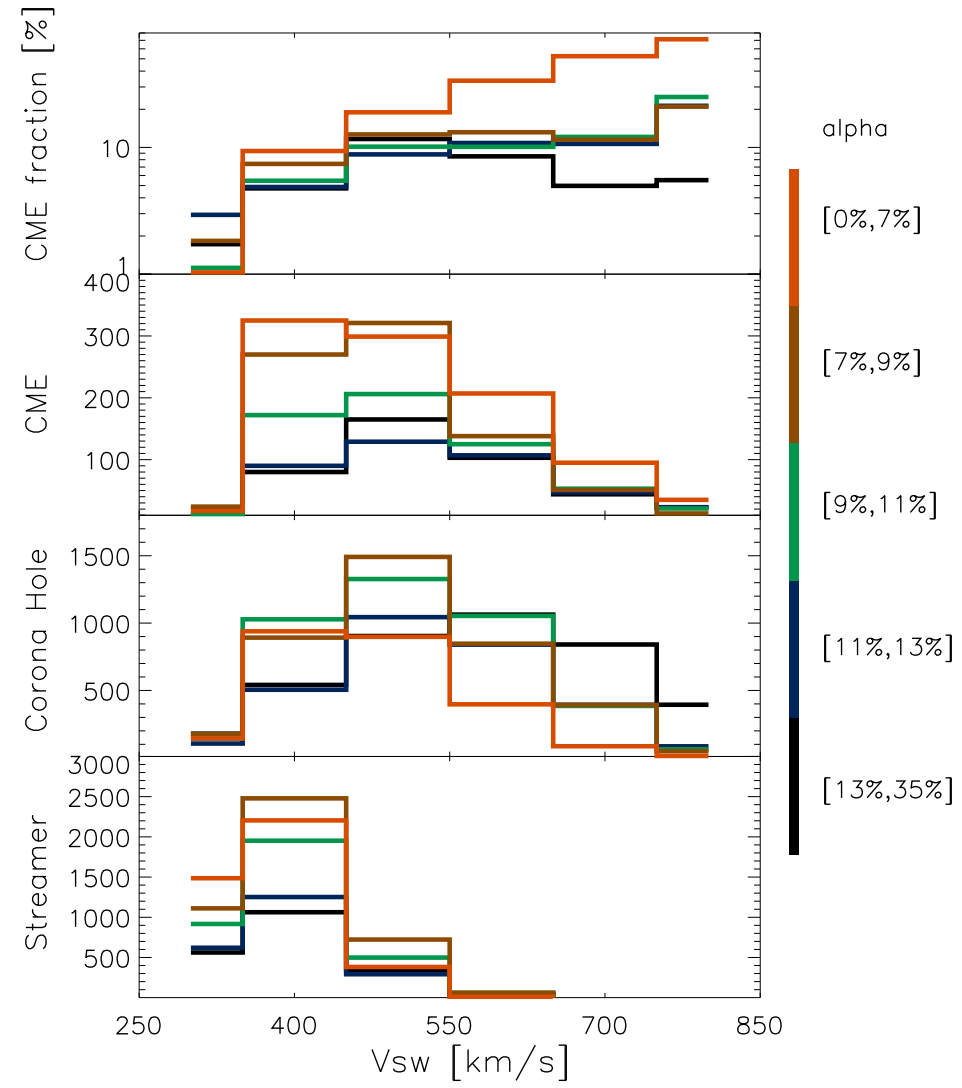

Fig. 8. Distribution of the three types of solar wind and the fraction of CMEs in the five alpha bins and six velocity bins.

However, $\mathrm{Si} / \mathrm{O}$ and $\mathrm{Mg} / \mathrm{O}$ are different from $\mathrm{Fe} / \mathrm{O}$ in that their abundance ratios do not decrease with solar wind speed even in the high alpha bins, i.e., for low solar activity. We may note that this analysis is a statistical work resulting from the samples including all types of solar wind, and results might be different from for a more restrictive data selection customary in typical abundance studies. The role of the magnetic effect cannot be neglected in considering this difference. Is it possible that the magnetic effect plays a more prominent role for $\mathrm{Si}$ and $\mathrm{Mg}$ than for Fe in the low solar magnetic activity bins? Our recent statistical 
study shows that iron displays higher charge states in fast wind than in slow wind (Wang et al. 2008), but for silicon and magnesium ions, the mean charge states basically decrease with the velocity ( $\mathrm{V}_{\mathrm{SW}}<750 \mathrm{~km} / \mathrm{s}$ ) in the low solar magnetic activity bins (Wang et al. 2008). If the low abundance ratio of $\mathrm{Fe} / \mathrm{O}$ in the fast wind when alpha is above 0.07 can be partly attributed to the high charge states of Fe in fast wind, we can understand the absence of a decrease in $\mathrm{Si} / \mathrm{O}$ and $\mathrm{Mg} / \mathrm{O}$ for fast wind in the low solar magnetic activity bins (more power resonating with $\mathrm{Mg}$ and $\mathrm{Si}$ than with $\mathrm{O}$ because of their lower charge states). In other words, due to wave-particle interactions, the increased ratio of charge states $\left(Q_{O} / Q_{S i}\right.$ or $Q_{O}$ / $Q_{M g}$ ) would result in a constant abundance ratio $(\mathrm{Si} / \mathrm{O}$ or $\mathrm{Mg} / \mathrm{O})$ in the fast solar wind.

The more prominent role of solar magnetic effects in fast solar wind may be due to the higher electron temperature powered by the MHD waves at the relative high altitude in the solar corona, i.e., at altitudes of 1.5-3 solar radii. Assuming that the fast solar wind is accelerated by ion cyclotron waves generated by the interaction and reconnection between open flux and small-scale closed loops at this height, ions are perpendicularly heated by the ion cyclotron waves and execute large gyro-orbits. Then density gradients in the flow can excite lower hybrid waves through which electrons then can be heated in the parallel direction (Laming \& Lepri 2007). Once heated by lower hybrid waves, the increased electron temperature would push the elemental ionisation to higher charge states. But things would be different in slow solar wind because the slow solar wind is believed to accumulate in closed loops for hours to days before it is released into the heliosphere (e.g. Uzzo et al. 2003). The ions experience sufficient heating in the loops and their velocity distributions become almost isotropic. The conditions for exciting lower hybrid waves might not be satisfied because of the disappearance of an-isotropic velocity distributions. Therefore, the charge states and the abundance ratios in slow and fast solar wind respond differently to the solar magnetic effects.

We also study helium, another high-FIP element. Helium does not show the similar trend as neon because He cannot be regarded as minor ion in the solar wind plasma and its fractionation may be associated with the bulk solar wind acceleration (see discussion in Aellig et al. 2001; Kasper et al. 2007).

In Fig. 9 we give an overview of the measured solar wind abundances underlying different solar magnetic activity bins. For comparison, we plotted the elemental abundances as a function of both FIP (right column) and mass (left column). The observed solar wind abundance ratios (relative to oxygen), compared to the photospheric abundance ratios, show the well-known FIP pattern. The abundance ratios as function of FIP can be grouped in three plateaus: a low-FIP plateau for elements with FIPs $<10 \mathrm{eV}$ which are the elements $\mathrm{Mg}, \mathrm{Fe}$, and $\mathrm{Si}$ showing the largest enrichments, separated by a step at about $10 \mathrm{eV}$ until about $20 \mathrm{eV}$ for the relative high-FIP elements including $\mathrm{C}$ and $\mathrm{O}$ with enrichments around 1. We mark the high-FIP element neon separately because it behaves different from $\mathrm{C}$ and $\mathrm{O}$. The highFIP element He often shows an abundance ratio of 0.5 (Aellig et al., 2001), i.e., it is depleted with respect to its photospheric abundance. The error bar marked on the low-FIP plateau represents the standard deviation of the average height of the low-FIP element group ( $\mathrm{Fe}, \mathrm{Si}$, $\mathrm{Mg})$.

In contrast to low-FIP (Mg, Fe, $\mathrm{Si}$ ) and high-FIP elements $(\mathrm{Ne}, \mathrm{He})$, no obvious variations with solar wind speed are found for the two high-FIP elements carbon and oxygen (oxygen 
$[\mathrm{X} / 0] /[\mathrm{X} / 0]$ photosphere
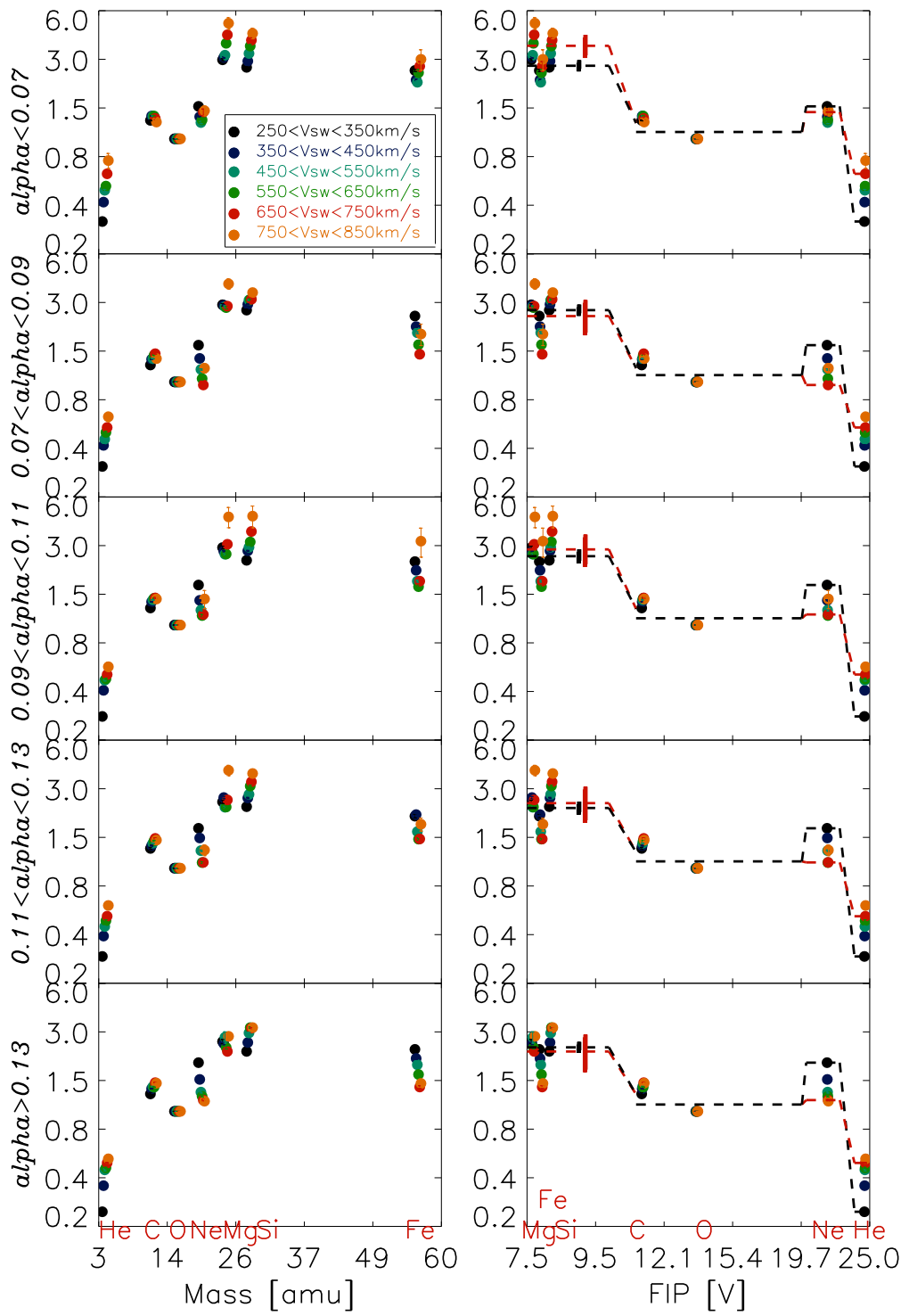

Fig. 9. Elemental abundance ratios (relative to oxygen) compared to photospheric ratios with different alpha, plotted as a function of FIP and mass. The FIP fractionation pattern basically shows three plateaus separated by a step at $10 \mathrm{eV}$ between the low-FIP elements (Mg, Fe, Si) and mid-FIP elements ( $\mathrm{C}$ and $\mathrm{O}$ ), $\mathrm{Ne}$ is marked separately from $\mathrm{C}$ and $\mathrm{O}$ because of its different behaviour, another step is observed between the high-FIP element $\mathrm{Ne}$ and He. The error bar marked on the low-FIP plateau represents the standard deviation of the average height of the low-FIP element group (Fe, $\mathrm{Si}, \mathrm{Mg}$ ). 
is used as reference). Carbon is slightly enriched relative to $O$, but this enrichment does not change significantly with the solar wind speed (see also Fig. 6). Therefore, the mid-FIP element plateau is plotted as a single plateau height that does not distinguish the fast wind from the slow wind. The average plateau height of low-FIP element group ( $\mathrm{Fe}, \mathrm{Si}, \mathrm{Mg}$ ) shows visible enhancement in the fast solar wind for high solar magnetic activity (alpha < 0.07), whereas it has no obvious variation in slow solar wind in all alpha bins. This suggests that mechanisms enhancing the upward transport of low-FIP elements should work equally well in low-speed CMEs and the ambient low-speed solar wind. The average enrichment factor for the low-FIP group (e.g. $\mathrm{Mg}$, Fe, $\mathrm{Si}$ ) relative to photospheric values is between 1.64 and 2.47 for low solar magnetic activity (alpha > 0.14), and it ranges from 2.78 to 4.15 when alpha is below 0.07 (large solar activity). For the high-FIP element $\mathrm{Ne}$, the plateau height for the low-speed solar wind is always higher than that for high-speed solar wind until alpha is below 0.07 , where they are close to each other. The plateau heights for Ne between different velocity bins can vary by as much as a factor of two. This is in contrast to the result in quasistationary solar wind, which is found to be constant and independent of solar wind type (e.g. von Steiger 1995; Geiss et al. 1994). But it agrees with the spectroscopically determined $\mathrm{Ne} / \mathrm{O}$ ratios in active regions and flares (McKenzie \& Feldman 1992) and it essentially agrees with the photospheric value (Anders \& Grevesse 1989). In 1992, Widing and Feldman demonstrated that the ratio $\mathrm{Mg} / \mathrm{Ne}$ in their spectroscopical observations can vary by as much as an order of magnitude, and its variation is correlated with the magnetic field morphology at the observed site on the solar surface. The largest $\mathrm{Mg} / \mathrm{Ne}$ values are found in polar plumes and diverging magnetic fields (Widing \& Feldman 1992). Although their report induced some arguments on effects of electron temperature or FIP (e.g. Doschek \& Laming 2000), in view of our finding that the dependence of the charge state ratios with solar magnetic activity is correlated with FIP, it is quite possible that the effects of electron temperature and FIP effects are supplementing each other for the elemental fractionation, for example, through redistribution of open magnetic flux on the solar surface, and excitation of ion cyclotron waves. Ions that are perpendicularly heated by ion cyclotron waves further excite lower hybrid waves through which electrons are heated (Laming \& Lepri 2007). Increased electron temperatures further influence the charge states of ions. Through wave-particle interactions, the modification of charge states would be finally reflected in elemental abundances.

\section{Conclusions}

In this paper, we present the influence of solar magnetic field activity on the charge state distributions and elemental abundances of heavy ions in the solar wind.

First, we observe a dependence of the charge state distribution of heavy ions with solar activity. This dependence is more important in the fast solar wind than that in the slow solar wind.

Second, iron is different from other species in that it displays lower charge states in slow wind than in fast solar wind.

Third, we find that the enrichment of low-FIP elements with respect to the photospheric values is around 2 when the fraction of the open magnetic flux on the visible side of the solar disc is above 0.14 . The elemental fractionation of low-FIP elements rises to $2.78-4.15$ 
when the fraction of the open flux is below 0.07, i.e., for high solar activity. These values are close to the enrichments of low-FIP elements with respect to the photospheric values in quasi-stationary fast- and slow solar wind, which suggests that the intrinsic mechanisms for the fractionation of stationary solar wind and intermittent solar wind might be similar.

Fourth, in the high solar magnetic activity (alpha < 0.07) case, the enrichment of heavy elements for the fast solar wind is well correlated with the high occurrence rate of CMEs in the solar wind. Both the charge state ratios and the elemental abundance ratios show less dependence on alpha in slow solar wind than in fast solar wind.

Fifth, The dependence of the charge state ratios on alpha is correlated with FIP, which implies an important role of solar magnetic field activity in the elemental fractionation of the solar wind. The possible linkage between solar magnetic field and elemental fractionation suggests the important role of the lower hybrid waves.

\section{Acknowledgements}

We thank the ACE SWICS-SWIMS instrument team and ACE Science Center for providing the ACE data. We thank SOHO and in particular the SOI. team at the Stanford University for making SOHO MDI data available. One of the author (Xuyu Wang) thanks Dr. Xuepu Zhao at Stanford University for providing the CSSS model and Dr. Yuzong Zhang in NOAC for help understanding the model. This work is supported by the National Natural Science Foundations of China (41074123), the National Basic Research Program of China (2006CB806303) and Y07024A900.

\section{References}

Aellig, M. R. et al. 1999, Solar Wind Nine, American Institute of Physics, 255

Aellig, M. R., Lazarus, A.J., \& Steinberg, J. T. 2001, Geophys. Res. Lett., 28, 2767

Anders, E., \& Grevesse, N. 1989, Geochim. Cosmochim. Acta 53, 197

Arge, C. N., \& Mullan, D. J. 1998, Sol. Phys., 182, 293

Arnaud, M., \& Raymond, J. 1992, ApJ, 398, 394

Bochsler,P., et al. 1998, ESA SP, 404, 37

Bochsler, P. 2007, Astron. Astrophys. Rev., 14, 1

Bogdan, Y. J., \& Low, B. C. 1986, ApJ, 306, 271

Bryans, P., Badnell, N. R., Gorczyca, T. W., Laming, J. M., Mitthumsiri, W., \& Savin, D. W. 2006, ApJS, 167, 343

Chen, J. 1996, JGR, 101, 27499

Chen, Y., Esser, R., \& Hu Y. 2003, ApJ, 582, 467

Doschek, G. A. 1983, Sol. Phys., 86, 9

Doschek, G. A. \& Laming, J. M. 2000, ApJ, 539, L71

Esser, R., \& Edgar, R. J. 2000, ApJ, 532, L71

Esser, R., \& Edgar, R. J. 2001, ApJ, 563, 1055

Feldman, U. 1992, Phys. Scr., 46, 202

Fisk, L.A., Schwadron, N. A., \& Zurbuchen, T. H. 1999, J. Geophys. Res., 104, 19765

Fisk, L. A. 2003, J. Geophys. Res., 108(A4), 1157, doi:10.1029/2002JA009284

Fisk, L. A., \& Zurbuchen, T. H. 2006, J. Geophys. Res., 111, A09115, doi: 10.1029/2005JA011575 
Geiss, J., et al. 1994, Eos Trans. AGU, 75, \#16, 278

Gopalswamy, N. et al. 2003, ApJ, 586, 562

H’enoux, J. C. 1998, Space Sci. Rev., 85, 215

Hildner,E. et al. 1976, Solar Physics, 48, 127

Kahler, S. W. 1992, Ann. Rev. in Astronomy and Astrophysics, 30, 113

Kartavykh, Y.Y., et al. 2007, ApJ, 671, 947

Kasper, J. C., Stevens, M. L., Lazarus, A. J., et al. 2007, ApJ, 660, 901

Klecker, B., et al. 2006, Adv. Space Res. 38, 493

Ko, Y.-K., Fisk, L.A., Geiss, J., Gloeckler, G., \& Guhathakurta, M. 1997, Sol. Phys., 171, 345

Ko, Y.-K., Gloeckler, G., Cohen, C. M. S., \& Galvin, A. B. 1999, J. Geophys. Res., 104, 17005, doi: 10.1029/1999A900112

Koutchmy, S. 1977, Solar corona, in Illustrated Glossary for Solar and Solar-Terrestrial Physics, edited by

A. Bruzek and C. J. Durrant, p. 39, D. Reidel, Nowell, Mass.

Laming, J. M. 2004, ApJ, 614, 1063

Laming, J. M., \& Lepri, S. T. 2007, ApJ, 660, 1642

Levine, R. H. 1982, Sol. Phys. 79, 203

Liewer, P. C., Neugebauer, M., \& Zurbuchen, T. H. 2004, Sol. Phys., 223, 209

Liu, Y. \& Hayashi, K. 2006, ApJ, 640, 1135

Marsch, E., von Steiger, R., \& Bochsler, P. 1995, A\&A, 301, 261

McKenzie, J. F. \& Feldman, U. 1992, ApJ, 389, 764

Meyer, J.-P., 1985, ApJS, 57, 151

Möbius, E., et al. 2003, Proc.28th Int. Cosmic Ray Conf. (Tsukuba) 6, 3273

Neugebauer, M., Liewer, P. C., Smith, E. J.,et al. 2002, J. Geophys. Res., 107, 1488,

doi:10.1029/2001JA000306

Owens, M. J., Schwadron, N. A., Crooker, N. U., Hughes, W. J., \& Spence, H. E. 2007, Geophys. Res. Lett., 34, L06104, doi: 10.1029/2006GL028795

Parker, E. N. 1958, ApJ, 128, 664

Poduval, B., \& Zhao, X.-P. 2004, J. Geophys. Res., 109(A8), A08102, doi: 10.1029/2004JA010384

Reames, D. V. 1999, Space Sci. Rev., 90, 413

Reisenfeld, D. B., Gosling, J. T., Forsyth, R. J., et al., 2003, Geophys. Res. Lett, 30(19), 8031

Richardson, I.G., \& Cane, H. V. 2004 J. Geophys. Res., 109(A9), A09104, doi: 10.1029/2004JA010598

Schatten, K. H. 1971, Cosmic Electrodyn., 2, 232

Schwadron, N. A., Fisk, L. A., \& Zurbuchen, T. H. 1999, ApJ, 521, 859

Scudder, J. D., \& Olbert, S. 1983, In JPL Solar Wind Five, 163

Sheeley, N. R., 1996, ApJ, 469, 423

Smith, E. J., \& Balogh, A. 1995, Geophys. Res. Lett., 22, 3317

Suess, S. T., \& Smith, E. J., 1996, Geophys. Res. Lett., 23, 3267

Uzzo, M.,Ko, Y.-K., Raymond, J. C., et al., 2003, ApJ, 585, 1062

von Steiger, R., Wimmer Schweingruber, R. F., Geiss, J., et al., 1995 Adv. Space Res., 15, 3

Wang, X., Klecker, B., \& Wurz, P., 2008, ApJ, 678, L145

Wang, Y. M. et al. 1998, ApJ, 508, 899

Widing, K. G. \& Feldman, U. 1992, ApJ, 392, 715

Wurz, P., et al. 1998, Geophys. Res. Lett., 25, 2557 
Wurz, P., et al. 1999, Phys. Chem. Earth(C), 24(4), 421

Wurz, P., Bochsler, P., Paquette, J. A., \& Ipavich, F. M., 2003a, ApJ, 583, 489

Wurz, P., et al. 2003b, Solar Wind X, American Institute Physics, 679, 685

Yashiro, S., et al., 2004, J. Geophys. Res., 109, Issue A7, CiteID A07105

Zhao, X.-P., \& Hoeksema, J. T., 1995, J. Geophys. Res., 100, 19

Zhao, X.-P., Hoeksema, J. T., \& Rich, N. B. 2002, Adv. Space Res., 29, 411

Zhou, G.-P., Wang, J. X., \& Cao, Z. L. 2003, A\&A, 397, 1057 


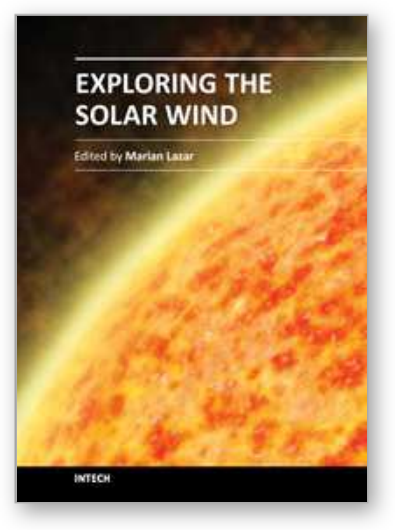

\author{
Exploring the Solar Wind \\ Edited by Dr. Marian Lazar
}

ISBN 978-953-51-0339-4

Hard cover, 462 pages

Publisher InTech

Published online 21, March, 2012

Published in print edition March, 2012

This book consists of a selection of original papers of the leading scientists in the fields of Space and Planetary Physics, Solar and Space Plasma Physics with important contributions to the theory, modeling and experimental techniques of the solar wind exploration. Its purpose is to provide the means for interested readers to become familiar with the current knowledge of the solar wind formation and elemental composition, the interplanetary dynamical evolution and acceleration of the charged plasma particles, and the guiding magnetic field that connects to the magnetospheric field lines and adjusts the effects of the solar wind on Earth. I am convinced that most of the research scientists actively working in these fields will find in this book many new and interesting ideas.

\title{
How to reference
}

In order to correctly reference this scholarly work, feel free to copy and paste the following:

X. Wang, B. Klecker and P. Wurz (2012). Solar Wind Composition Associated with the Solar Activity, Exploring the Solar Wind, Dr. Marian Lazar (Ed.), ISBN: 978-953-51-0339-4, InTech, Available from:

http://www.intechopen.com/books/exploring-the-solar-wind/solar-wind-composition-associated-with-the-solaractivity

\section{INTECH}

open science | open minds

\section{InTech Europe}

University Campus STeP Ri

Slavka Krautzeka 83/A

51000 Rijeka, Croatia

Phone: +385 (51) 770447

Fax: +385 (51) 686166

www.intechopen.com

\section{InTech China}

Unit 405, Office Block, Hotel Equatorial Shanghai

No.65, Yan An Road (West), Shanghai, 200040, China

中国上海市延安西路65号上海国际贵都大饭店办公楼 405 单元

Phone: +86-21-62489820

Fax: +86-21-62489821 
(C) 2012 The Author(s). Licensee IntechOpen. This is an open access article distributed under the terms of the Creative Commons Attribution 3.0 License, which permits unrestricted use, distribution, and reproduction in any medium, provided the original work is properly cited. 